\title{
The Cotwin Method and Experimental Science
}

\section{THE COTWIN METHOD AND EXPERIMENTAL SCIENCE}

\section{RUNE CEDERLÖF}

Department of Environmental Hygiene, The Karolinska Institute, Stockholm, Sweden

A strict evaluation of cause and effect has for long been regarded to require the tools of experimental design. The main approach is to constitute two or more subject groups by randomization or equating procedures, whereupon one or more groups will be manipulated by the experimenter, whilst one groups is left unmanipulated and thus serves as a control group. There are numerous experimental designs aimed at quantifying the effect of manipulation, one of which is the cotwin control method. The advantage of the method is the minimizing of the intrapair variance by the genetic identity between the members of a pair.

Experiments, however, have limitations in problems where the presumed causal factor cannot be manipulated but exists in certain population groups and not in others. In comparing such groups the method of randomization in its true sense cannot be employed. Even matching or equating may cause bias because the variable or variables which induced the presumed causal factor in certain individuals may not be known or measurable and are thus not equatable.

In such cases a modified cotwin method may be used, which employs twins where the one member has the presumed causal factor and his cotwin not. The results of a comparison between the members of such discordant pairs will not be biased by genetic differences that may be correlated to the presumed causal factor. The greater resemblance between the twins in a pair in early environmental influences will further enhance the reliability of the method.

This application of the cotwin method is of great value in epidemiological research in connection with evaluation of the effect of environmental exposures such as air pollution, tobacco smoking, etc. It has been applied in this sense in Denmark, USA, and Sweden, and examples will be given.

Prof. Rune Cederlöf, Department of Environmental Hygiene, The Karolinska Institute, S-I04 OI Stockholm, Sweden

\section{ALCOHOL CONSUMPTION IN RELATION TO VARIABLES ASSOCIATED WITH ISCHEMIC HEART DISEASE}

\section{A Cotwin Control Study}

\section{MARTEN MYRHED, BIRGITTA FLODERUS}

Medical Clinic, Serafimer Hospital, Stockholm, Sweden Department of Environmental Hygiene, The Karolinska Institute, Stockholm, Sweden

The purpose of the study was to investigate the relation between a moderate alcohol consumption and variables associated with IHD when genetic factors were under control. Seventy alcohol-discordant male twin pairs, aged $45-65$, have been examined. Significantly higher systolic and diastolic blood pressures were found in the highalcohol group (HAG) with respect to the low-alcohol group (LAG). A significantly higher number of cigarettes/day were smoked in the HAG, in which group cough, phlegm, asthma, and dyspnea, were significantly more frequent than in the LAG. Serum cholesterol $>200 \mathrm{mg} \%$ was significantly more prevalent in the HAG, while no differences could be shown for triglyceride level and serum lipoproteins. Significant differences as to raised level of serum uric acid, fasting blood glucose, and a pathologic glucose tolerance, were also found in the HAG. The importance of these variables for development of IHD in groups, where the constitutional factors are kept under control, is yet not known. It is assumed that the results might imply earlier IHD manifestations in the high- than in the low-alcohol group.

M. Myrhed, M.D., Serafimerlasarettet, Fack 12 700, 11283 Stockholm, Sweden 